Islamic Heritage Architecture and Art II 149

\title{
THE VOCABULARY OF PERCEPTION AND DESIGN OF ISLAMIC GEOMETRIC PATTERNS
}

\author{
MOHAMAD NASRI \\ Faculty of Architecture and Design, City University, Tripoli, Lebanon
}

\begin{abstract}
This paper introduces a methodological distinction between three different scholarly interpretations of the forms and meanings of geometric ornaments in our heritage of Islamic art and architecture: an external cultural position, an esoteric religious argument, and an internal scientific approach. The major part of the paper is then directed beyond cultural allegiances or prescriptions of the Islamic faith and revolves instead around the internal formalistic and purely aesthetic aspects of reconstructing and making of geometric patterns, with the aim of exploring their vocabulary of perception, and their generative principles and inherent processes. The analysis starts at the very basic level where geometric patterns can be perceived as packing of open or enclosed surface polygons or linear configurations. Other means of perception relate to polygons' apparent and inherent geometry, tone or colour, and the application of a quasi-third dimension either through figure-ground reversal or by perceiving linear designs as interlocking elements beyond the $2 \mathrm{~d}$ plane. The paper then investigates the relationship between means of visual perception and the inherent repetition, geometry and symmetry of patterns on the level of constituent polygons, repetitive tiles, and the design as a whole. Beyond the narrow meanings of likeness and identity in bilateral symmetry, alternative concepts of symmetry are introduced and then applied in setting up a comprehensive vocabulary of $2 \mathrm{~d}$ geometric patterns based on the classification discovered by crystallographers and developed by mathematicians. The paper concludes by demonstrating the power of combining geometric and symmetry systems in recreating traditional designs or generating new patterns.
\end{abstract}

Keywords: geometric patterns, vocabulary of perception, generative principles, symmetry operations.

\section{INTRODUCTION}

In recent years, an increased effort has been expended in the study of the inherent design, the meaning, and the historical and cultural context of Islamic ornamental patterns. Within a cultural argument, they are often framed into social products or historical styles [1]. In the esoteric explanation, on the other hand, theological and mystical principles are used as interpretive tools. Symbolism is given an esoteric dimension, and formal and structural order becomes a manifestation of the principle of 'unity' [2], [3]. The rational/positivistic approach, finally, disregards the question of meaning in the study of Islamic ornament, whether socially related or more particularly bound by the faith, and concentrates instead on the formalistic aspects of the design and its geometric and mathematical processes [4].

The scope of my interest here goes beyond the cultural allegiances or prescriptions of the Islamic faith; it rather revolves around the internal formalistic and purely aesthetic aspects of the art of making of geometric patterns, with the aim of exploring their vocabulary of perception, and their inherent geometric principles.

\section{THE VOCABULARY OF PERCEPTION}

The design of a single $2 \mathrm{~d}$ repeating pattern can be communicated and perceived in different ways using basic graphic vocabulary like line and tone in order to express the geometry and symmetry systems inherent within the design. A multitude of readings, and consequently a variety of patterns, will result from different applications of the vocabulary of line and tone on the same design. 
On the very basic level, geometric patterns can be perceived as packing of open or enclosed surface polygons or linear formations. Introducing tone or color to surface polygons or even to linear elements is another means of perception. An additional example is the addition of a quasi-third dimension either through figure-ground reversal of filled polygons, or by perceiving linear designs as interlocking elements beyond the two dimensions of the plane. This section will explore these and other examples investigating the relationship between means of visual perception and the inherent geometry and symmetry of patterns. This will set the stage for the following section that deals with systems of symmetry and repetition.

\subsection{Surface polygons}

This reading acknowledges the primary nature of two-dimensional repeating patterns as surface-filling designs. The surface is seen as edge-to-edge packing of closed polygons (Fig. 1(a)). In some cases, open polygonal areas play a similar role of filling space in infinite riblike or band-like configurations (Fig. 1(b)).

Another way of distinguishing polygon types relates to whether polygon edges are either curved or rectilinear (Figs 2(a) and 2(b)). Patterns within the scope of this study are mostly rectilinear and based on closed polygons. A third distinction relates to the internal axial or rotational symmetry of the polygon (Figs 2(c) and 2(d)). Still another equally important distinction is the geometric proportioning system on which the polygon is based. Notice the common geometric system in both rectilinear and curved cases (Figs 2(e) and 2(f)).

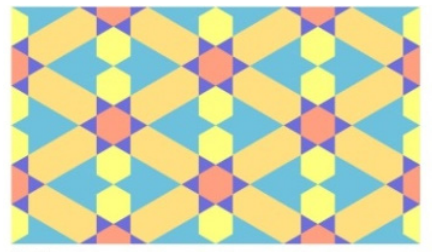

(a)

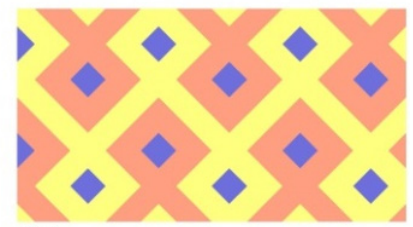

(b)

Figure 1: Surface filling polygons. (a) Closed polygons; (b) Open polygonal bands.

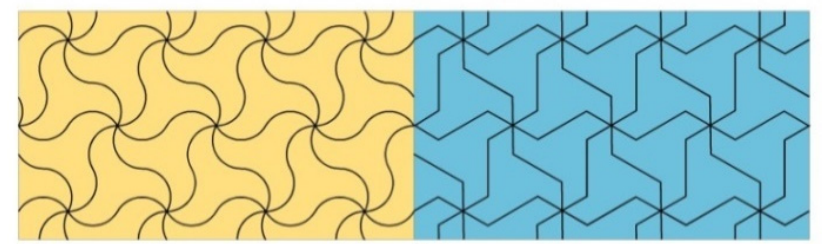

(a)

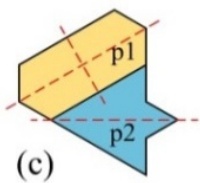

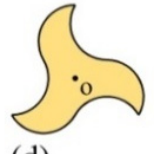

(d) (b)

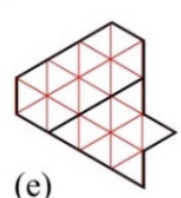

(e)

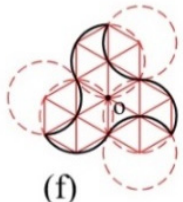

(f)

Figure 2: Polygon geometry. (a) and (b) Curvilinear vs. rectilinear polygons; (c) Internal axial symmetry; (d) Rotational symmetry; (e) and (f) Geometric proportioning. 
Every pattern is ultimately made out of a limited number of fundamental polygon types repeated in $2 \mathrm{~d}$ space. This important characteristic is illustrated in Fig. 3(a) using the same pattern of Fig. 1(a). Different types of polygon repeat in different quantities and the proportions of constituent polygon types within the design as a whole turn to be relevant. And since a pattern is made out of repetitive modules, then the number of occurrences of each polygon type within a repeating unit becomes also important (Fig. 3(b)).

\subsection{Mass-void reversal}

Once we are able to perceive patterns as space-filling surface-polygons, the reversed reading of voided polygons that leave empty space behind is also possible. The phenomenon becomes interesting when both filled and empty polygons combine in an orderly manner in one massvoid reading of a single design.

Any closed-polygon pattern, whose filled-polygon reading conforms to interlace rules (explained in 2.4 below) can be perceived as alternating concentric polygon sets that follow an on-off logic of figure-ground reversal (Fig. 4(a)). Also, mass-and-void, black-and-white or light-and-dark alternative themes can be conceived of here. The same also holds true for some rib or band-like designs based on open polygons as in Fig. 4(b). In this example, reversing the dark and light areas results in a translated copy of the same design.

\subsection{Fundamental linear polygons}

This reading of a geometric ornament reduces the design into an arrangement of straight-line configurations in $2 \mathrm{~d}$ space. In relation to the filled polygon reading, lines represent the shared edges of neighboring polygonal surfaces of the design. But in a linear reading, we start to perceive the lines independently from the polygons that they define. The eye now might start at a certain point and follow a linear element across different polygons until it either appears to be infinite as in Fig. 5(a) or it circles back to its origin as in Fig. 5(b).
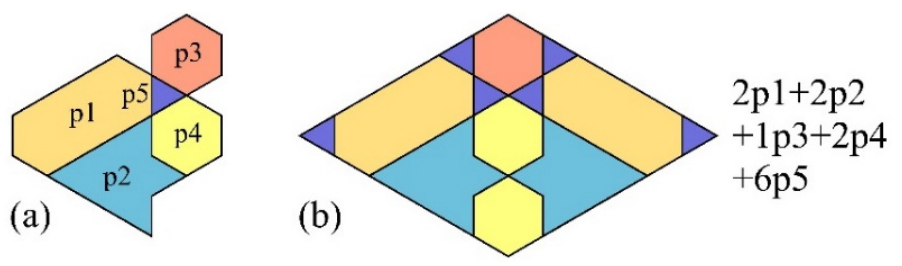

Figure 3: Polygon types and repetition. (a) Fundamental polygons; (b) Polygons of a repeating unit.

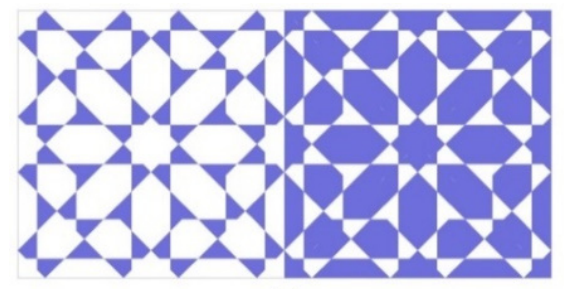

(a)

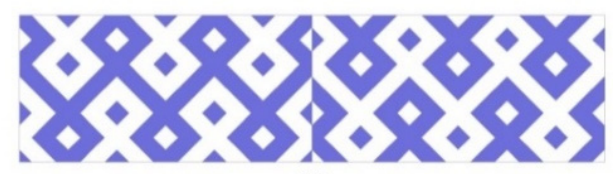

(b)

Figure 4: Mass-void reading. (a) Closed polygons; (b) Open polygonal bands. 


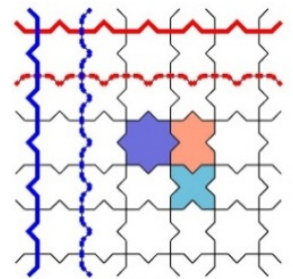

(a)

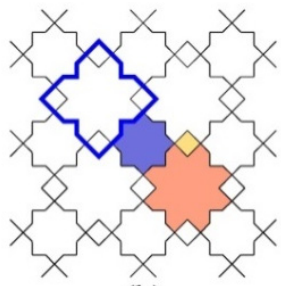

(b)

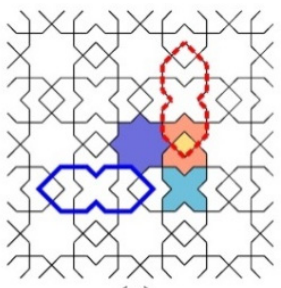

(c)

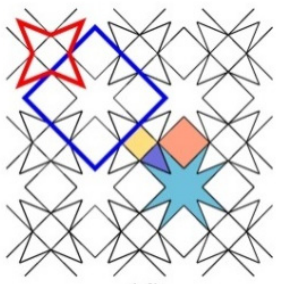

(d)

Figure 5: Linear reading. (a) Fundamental linear configurations; (b) A single linear polygon; (c) Two reflected linear polygons; (d) Two distinct linear polygons.

The reading becomes more interesting when the whole pattern starts to be perceived as an interlocking web of such linear elements which are essentially either endless continuous lines or closed linear polygons. In many cases, the repetition of a single linear element, maintaining the same orientation, as in Fig. 5(b), or with different orientations, as in Figs 5(a) and 5(c), suffices for generating the design. In other cases, 2 or more distinct elements play a similar role as in Fig. 5(d). Perceiving a pattern as interlocking linear elements is particularly possible in designs that conform with interlace rules explained below.

\subsection{Interlace designs}

Interlace designs are essentially based on a linear perception of geometric patterns but they also embody properties from surface and mass-void readings (Figs 6(a1), (b1) and (c1)). When, in a linear pattern, linear elements start to assume a certain width, i.e. linear surface, the perception of interlocking lines changes into a perception of interlacing surface elements that overlap to produce a new reality of the design ( Figs 6(a2), (b2) and (c2)). These elements repeat in an array of infinite broken lines as in Fig. 6(a2), or finite closed linear polygons as in Fig. 6(b2). Their developed width gives them a surface of their own until there comes a time when it is difficult to differentiate between a surface and a linear reading of the design as in Fig. 6(c2).

Interlace works in an on-off sequential fashion: if one linear element overpasses a second element, the next time it encounters a third element it should underpass it (Figs 6(a2), (b2) and (c2)). The overpass-underpass logic can be emphasized by a system of alternating colors (Fig. 6(c2)). Interlace designs can only be developed from a linear pattern when no more than 2 lines meet or cross at any one point. Interlace designs can only be developed from a filledpolygon reading of a pattern when all polygon vertices are only shared by 2 or 4 polygons; refer to vertices V2 and V4 in Figs 6(a1), (b1) and (c1).

Interlace designs can be subjected to foreground-background reversal or read as massand-void, black-and-white, or light-and-dark (Figs 6(a3), (b3) and (c3)). Interlace elements, decomposed into their finite closed polygon components, can be applied or embossed on a background surface material but can be less easily conceived of as perforated screens.

\subsection{Lace designs}

If a pattern of linear elements does not conform to interlace rules it can still be transformed into what might be called a lace design as illustrated in Figs 7(a) and (b). The difference here is that the linear elements have width but they simply meet each other without interlocking. 


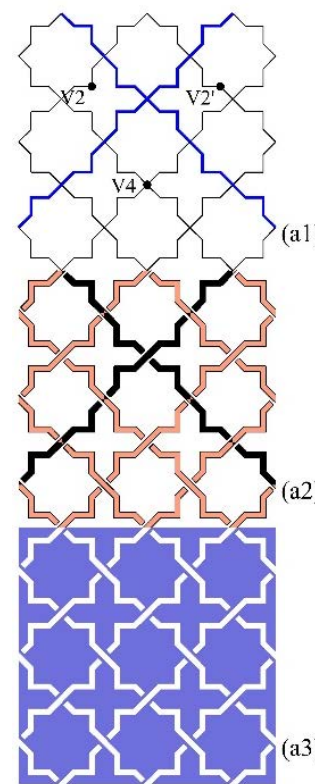

(a)

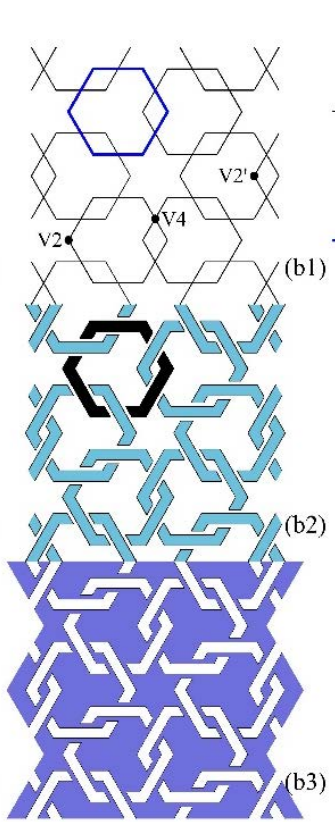

(b)

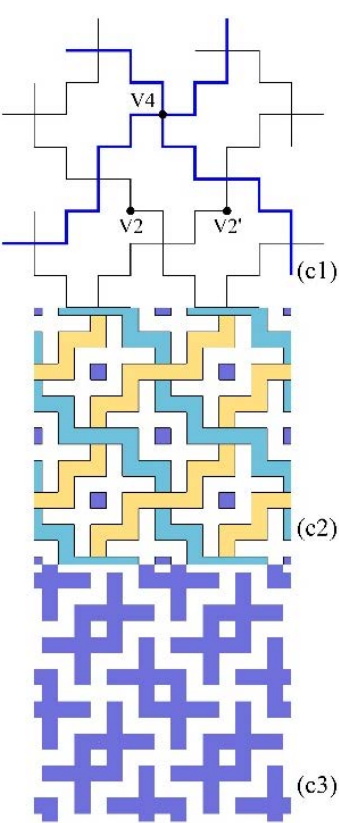

(c)

Figure 6: Interlace reading. (a) Infinite interlace elements; (b) Closed interlace polygon; (c) Interlace elements assuming maximum surface.

(a)

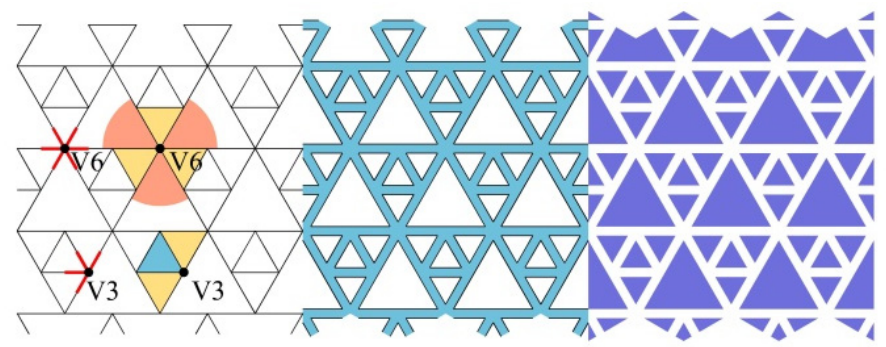

(b)

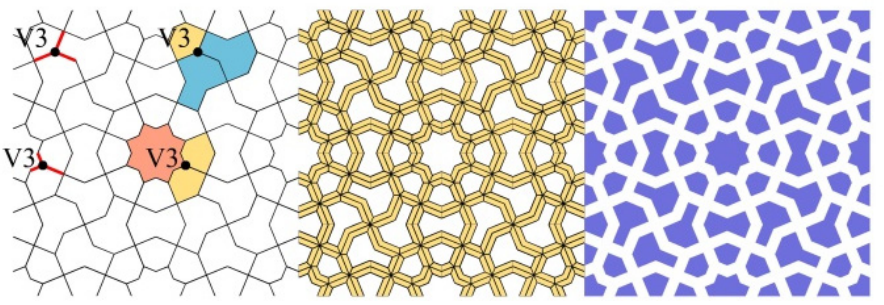

Figure 7: Lace design.

They cannot interlace in a continuous up-down fashion because it is enough to have at least one point at which more than 2 lines either meet or cross each other to disrupt the whole system as shown at points V3 and V6 in Fig. 7. 
Lace designs can be easily perceived in a foreground-background relationship or as massand-void, black-and-white, or light-and-dark. The mass-void relationship can be directly applied in terms of architectural and interior design elements like partition walls, or window screens for privacy or for filtering of natural light.

\subsection{Fundamental repetitive units}

This is a reading of geometric patterns that is less concerned with individual polygonal elements, and more in favor of conceiving of larger modules of the design that appear to generate the whole by endlessly repeating in $2 \mathrm{~d}$ space. This involves a shift from the local symmetry of a single polygon to symmetry of a larger module and ultimately to global symmetry of the design as a whole. A clearer view of the part-whole structure of the design would follow. The design is seen as a tessellation of different fundamental units, each of which can be physically conceived of as a repetitive tile.

When dealing with repetition different attributes of a pattern can be selectively considered: Polygon geometry, surface tone or color, and interlace or in-fill details. Added to that, there is always more than one way of conceiving of a repetitive tile within the same pattern depending on the choice of size, boundary shape, and type of repetition. A tile can be selectively reduced to the minimal size needed to regenerate the pattern as in modules $\mathrm{C} 1$, C2, C3 and C4 in Fig. 8(b). Bigger and bigger tiles are always possible. A tile can either have a regular boundary that cuts through polygons in order to maintain a regular geometry of its own, as in Fig. 8(b), or a less regular boundary that follows polygon edges, as in Fig. 8(c). And finally, a tile can be delineated according to different preconceptions of systems of repetition whether based on simple translation or other modes of reflection and rotational symmetries as will be explained in the next section.

It is important to note here that alternative ways of conceiving of a module will directly affect methods of designing new patterns and will ultimately direct manufacturing processes of physical tiles that are meant to cover a real surface. In the latter case, the best tiles can be those with boundaries that do not cut through edges of individual polygons, and yet maintain the most regular shape possible.

\section{THE VOCABULARY OF SYMMETRY}

In discussing alternative means of perceiving geometric patterns, we have found that symmetry and geometry play a major role in perceptual distinctions made on the level of constituent polygons, repetitive tiles, as well as the design as a whole. It is time now to explore in more depth alternative means of achieving space filling in an orderly manner, i.e. conceiving of orderly systems of repetition by combining different modes of symmetry.

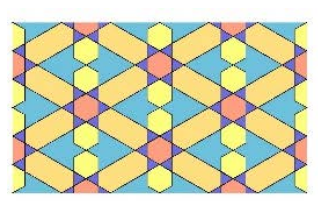

(a)
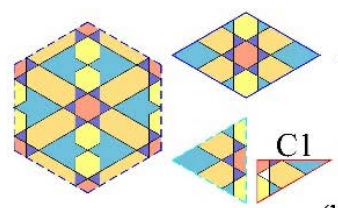

(b)

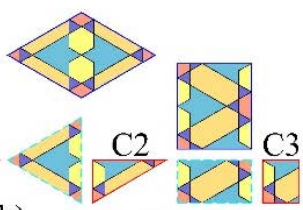

$\mathrm{C} 4 \square$

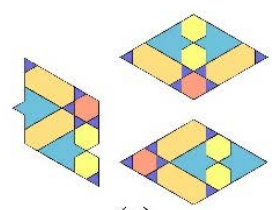

(c)

Figure 8: Modular repetition. (a) A 2d repeating pattern; (b) Alternative repeating modules; (c) Polygonal tiles. 


\subsection{Symmetry operations}

Symmetry is possessed by any system if the system remains essentially unaltered after one or more repetition operations have been performed on it. It is evident that the repetition of any fundamental unit of a $2 \mathrm{~d}$ geometric pattern, whether linear, polygonal, or tile-like, is based on translation, reflection or rotation. Such processes appear to play the role of symmetry operations in the sense that the design as a whole does not alter when translated, reflected or rotated (depending on which symmetry type it possesses in the first place).

The simple 2d geometric pattern in Fig. 9 illustrates the notion. The pattern is infinite and based on a triangular grid. Suppose we highlight a selected L-shaped area $\mathrm{P}$ on a transparent copy of the design. After overlaying the copy on top of the base pattern, we can move it around in different ways and to different locations (P1, P2 and P3) while keeping both infinite patterns perfectly superimposed. Available moves that satisfy this condition are actually either specific linear translations, flipping the whole pattern along selected axes, or rotation around selected points.

Translation in this particular design is possible in multiples of either distance $\mathrm{d} 1$ or $\mathrm{d} 2$ (Fig. 10). In both cases translation is possible along three different axes and in opposite directions along each axis. If we shift or copy the whole design, or part of it, along $\mathrm{d} 1 \mathrm{or} \mathrm{d} 2$, and in any of the indicated directions, it will remain unaltered. Reflection in Fig. 11 works in a different way. The axes along which we can flip the pattern are actually bilateral axes of symmetry i.e. mirror-lines. Notice how the semi-regular hexagon is mirrored when reflected about the chosen vertical axis " $m$ ". A more complex type of reflection also exists along another inclined axis "g" connecting the centers of semi-regular hexagons. Notice how the selected triangles on the left side of "g" appear to be mirrored and translated into their reflected images on the right. This multiple process of translation and mirror reflection is called glide reflection.

Axes of reflection are therefore either mirror-lines highlighted as bold and continuous, or glide-lines as bold and dashed. Upon closer observation, it will be evident that two types of mirrors, $\mathrm{m} 1$ and $\mathrm{m} 2$, and two types of glides, $\mathrm{g} 1$ and $\mathrm{g} 2$ contribute to the grid of axes of reflection in this type of pattern.

Rotation, on the other hand, is based on repetition around specific points that play the role of centers of rotation (or roto-centers for short). When a design is rotated around a rotocenter, the design as a whole remains unchanged and would thus assume rotational symmetry. To illustrate rotational symmetry, two new designs are used in Fig. 12 and Fig. 13. In Fig. 12, three different types of rotation coexist: 2-fold, 3-fold, and 6-fold. In Fig. 13, 2-fold and

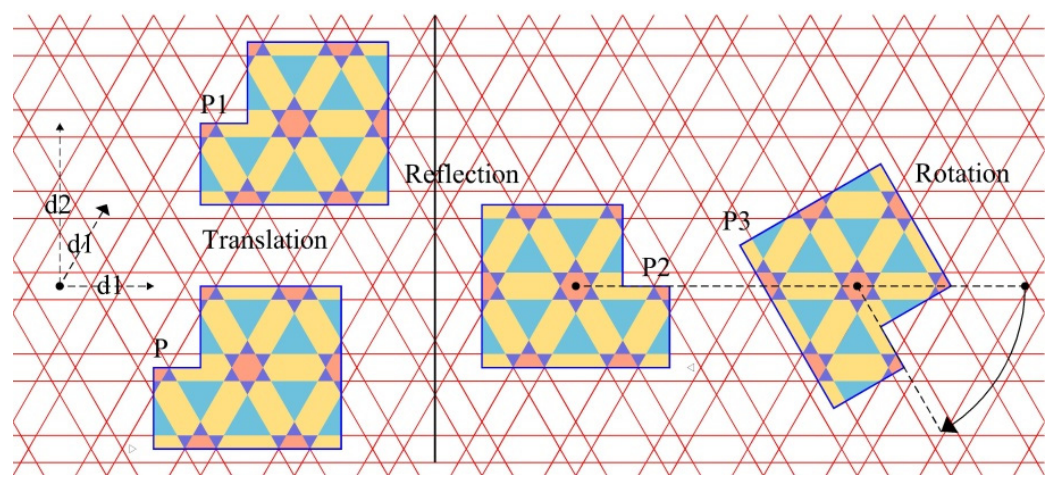

Figure 9: Translation, reflection and rotation. 


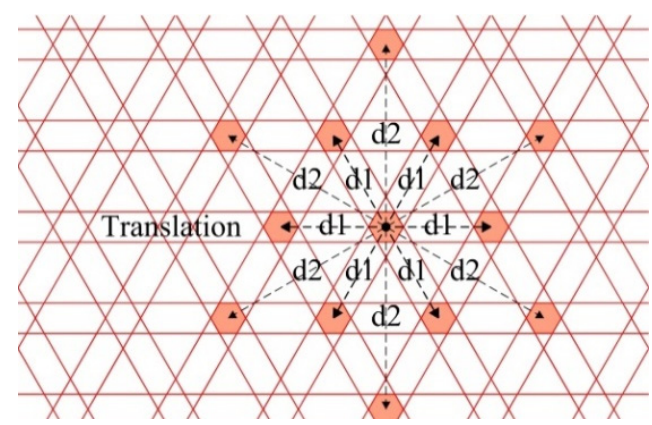

Figure 10: Translation.

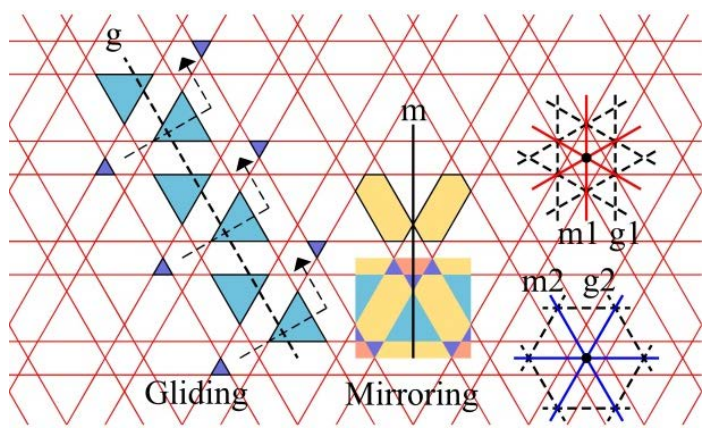

Figure 11: Reflection.

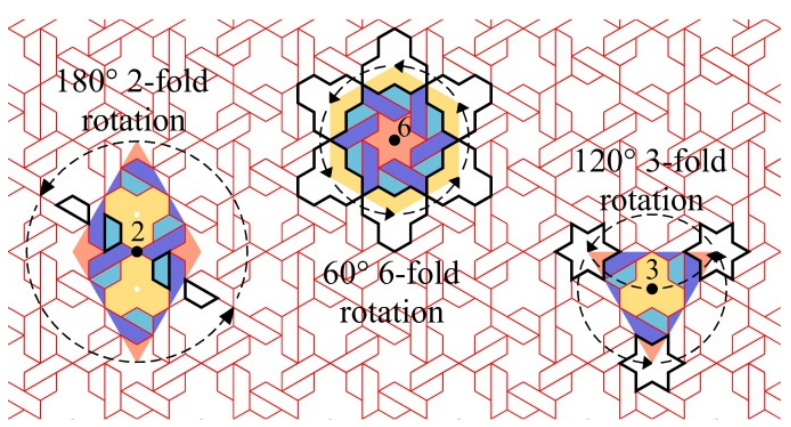

Figure 12: 2, 3, and 6-fold rotation.

4-fold rotations are possible. No other n-fold rotations are generally possible in any $2 \mathrm{~d}$ planar pattern. The reason is that only 2, 3, 4 or 6 -fold rotations are compatible with repetitive systems that cover the plane with no gaps. These systems use grids that are primarily based on the rectangle, the equilateral triangle, the square and the hexagon.

Two-fold rotational symmetry is not to be confused with bilateral mirror reflection. It involves 180-degree rotations, or multiples of that, around a 2-fold roto-center. Notice in Fig. 12 and Fig. 13 how the highlighted filled areas and the selected linear polygons repeat twice 


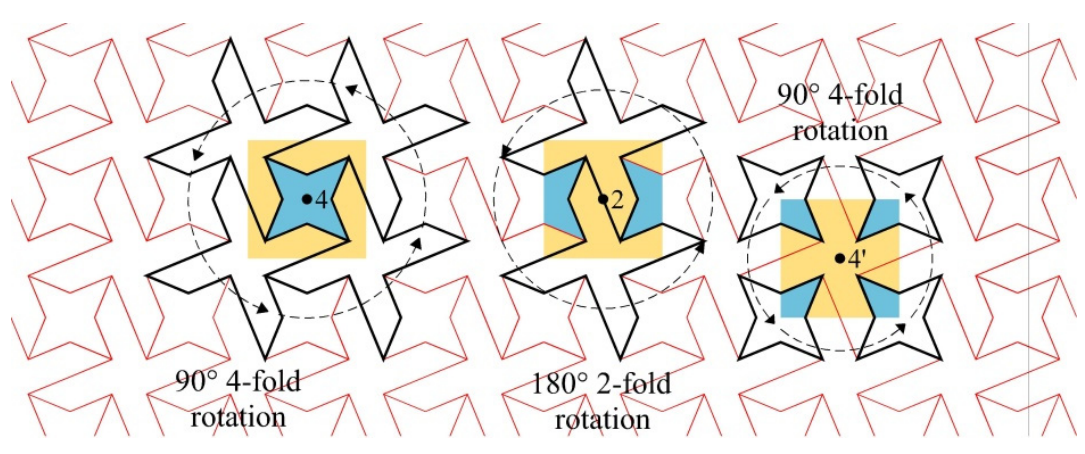

Figure 13: 2 and 4-fold rotation.

while rotating around the specified 2-fold centers. Each and every point that belongs to such areas or polygons undergoes the same 180-degree rotation while repeating.

Similar processes hold true in case of 3,4 and 6-fold rotations in Fig. 12 and Fig. 13. In three-fold rotation, the design, or any linear or planar part of it, can be rotated around the corresponding center 120, 240 or 360 degrees without being altered. Notice that in Fig. 13, there are 2 types of 4 -fold centers 4 and $4{ }^{\prime}$. The first is located at the center of the 4-pointed star, and the second at the center of the 4-pointed swastika. Rotations of 90, 180, 270 and 360 degrees are possible around both centers. Similarly, rotations of 60, 120, 180, 240, 300 and 360 degrees are also possible around the 6-fold centers in Fig. 12.

Roto-centers as we have seen above are specific points in a given $2 \mathrm{~d}$ pattern where rotational symmetry occurs. This actually applies in 2, 3, 4, and 6-fold rotational symmetries. If we consider however the general case of rotating a full turn of 360 degrees as 1-fold rotational symmetry, then this kind of rotation can occur at any point in a given pattern rather than at specific points in the design. One-fold rotation is therefore theoretically possible and will be practically indispensable in characterizing a particular group of $2 \mathrm{~d}$ patterns, as we will see below. In graphic illustrations a small filled circle, along with the number 2, 3, 4 or 6 next to it, will identify these centers (Fig. 14).

\subsection{Symmetry groups}

When applied on a certain $2 \mathrm{~d}$ design, different combinations of symmetry operations i.e. translation, reflection and rotation can generate an infinite variety of repeating $2 \mathrm{~d}$ geometric patterns. Groups of different patterns appear to have common characteristics in terms of the types and method of combination of these symmetry operations. This leads to the ability of classifying repeating patterns into a finite number of symmetry groups. The number of possible symmetry groups is 17 which coincides with the crystallographic and symmetry group theories that define 17 possibilities of planar symmetry first classified by the Russian scientist Fedorov [4]. Translation, reflection and rotational symmetry are at the core of these groups. Translation symmetry along different directions of the plane is common to all

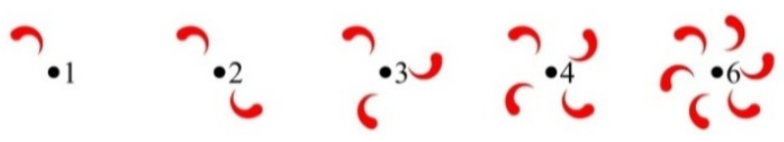

Figure 14: Roto-center types. 
repeating patterns and is the essence of their repeating modules, but although it is possible to classify patterns according to characteristics of their translating modules, rotational symmetry, on the other hand, appears to be more generic in categorizing these patterns in 5 broad categories based on rotational processes that are effective in covering the plane. These are the 1, 2, 3, 4 and 6-fold families (Fig. 15). When mirror and glide reflections are considered, these families manifest themselves in different sub-families or symmetry groups that sum up to the total of seventeen.

In Fig. 15 each symmetry group is identified by 2 types of labels. The first one simply identifies the rotational family and its order within the family ( $2 \mathrm{a}$ or $4 \mathrm{c}$ for example). The second type is more complex and summarizes the roto-center types and the mirrors and glides if any, plus the relative positioning between roto-centers and the mirrors or glides. An example is $4 \mid \mathrm{mg} 4$ ' $\mid \underline{2}$ where 4 and 4 " are the two 4 -fold roto-centers, and " 2 " is the only 2 -fold roto-center; underlined because it sits on a mirror line. The vertical line symbols after 4 and $4^{\prime}$ indicate that these centers belong to glide lines. The letters $\mathrm{m}$ and $\mathrm{g}$ between 4 and $4^{\prime}$ show that 4 and $4^{\prime}$ are mirrored as well as glided images of each other.

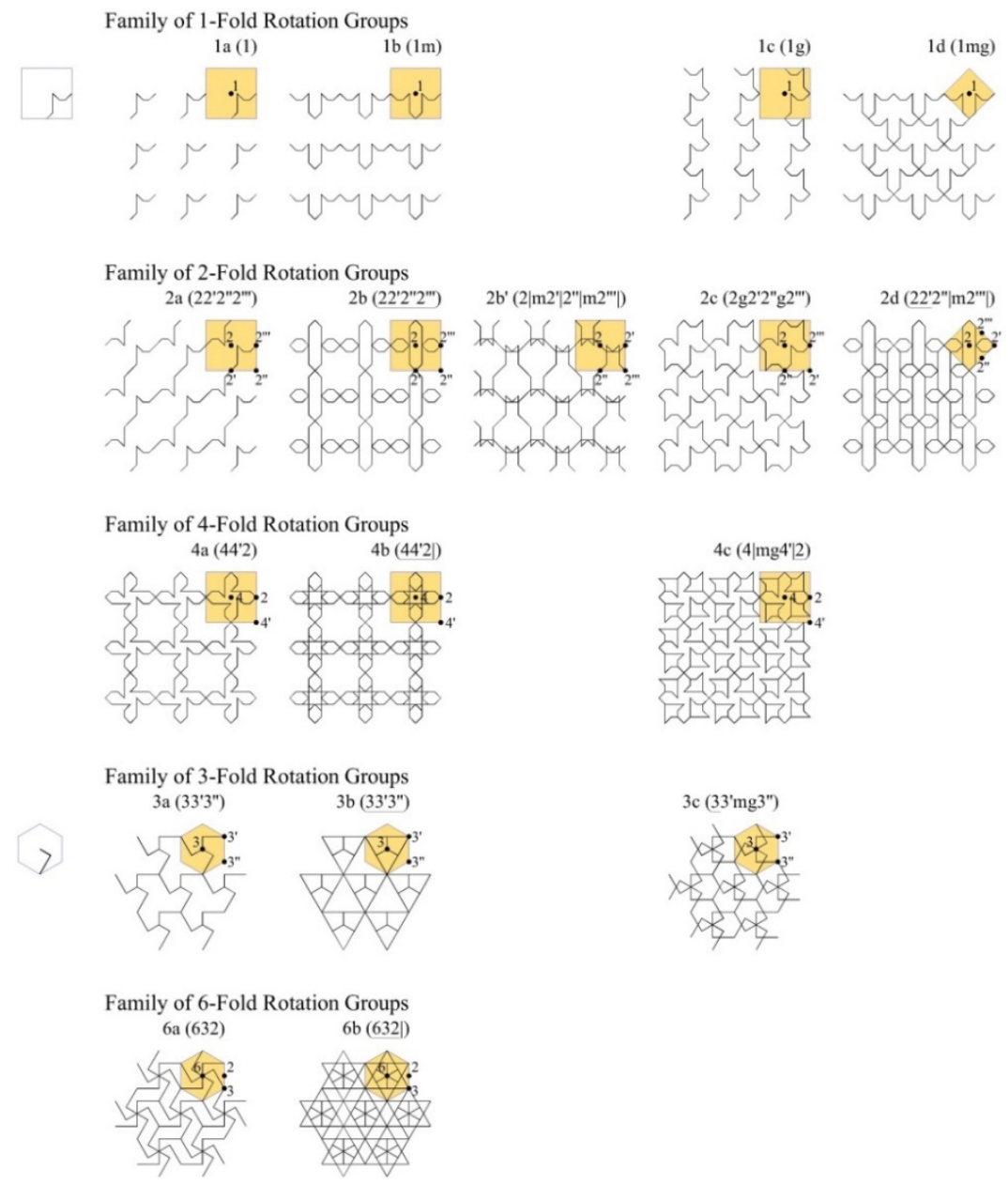

Figure 15: The 17 exclusive symmetry groups. 
Therefore, in terms of symmetry systems of repetition, there are only 17 ways of perceiving of $2 \mathrm{~d}$ geometric patterns. Owing to their abundance and infinite variety, the Islamic geometric patterns seem to exemplify most, if not all, of the 17 symmetry types.

\subsection{Applications in pattern generation}

The use of geometric tools and symmetry structures can serve in the analysis of historical ornamental patterns in the Islamic culture, and in the development of design rules and strategies for the production of new patterns. At the end of this short paper I will briefly illustrate the generative process of some geometric patterns in accordance with symmetry group theory and the vocabulary of repeating modules. First let us differentiate between three types of repeating modules: the repeat unit, the unit cell and the linear unit cell.

A repeat-unit (RU) is the smallest parallelogram (including rectangle and square) or hexagonal part of the design which when regularly translated in both directions of the plane would recreate the whole $2 \mathrm{~d}$ pattern (Figs 16(a) and (b)). A unit cell (UC) is the smallest surface part of a repetitive pattern that can generate the whole design under one or more symmetry operations of translation, reflection or rotation. These are the highlighted triangular areas within the above hexagons and squares. A linear unit cell (LUC) is the smallest part of a linear repetitive pattern that can generate the whole design under translation, reflection or rotation. These are the linear segments within the unit cells.

Different geometric systems may be chosen for the structuring of the hexagonal or square repeat units as shown in the left columns in Figs 16(a) and (b) [5]. Linear unit cell designs are then created by tracing on selected lines of the geometric structure within unit cells. The resulting designs are then repeated within repeat units in accordance with the specific rules of translation, reflection and rotation; 6-fold rotation in Fig. 16(a) and 4-fold rotation in Fig.
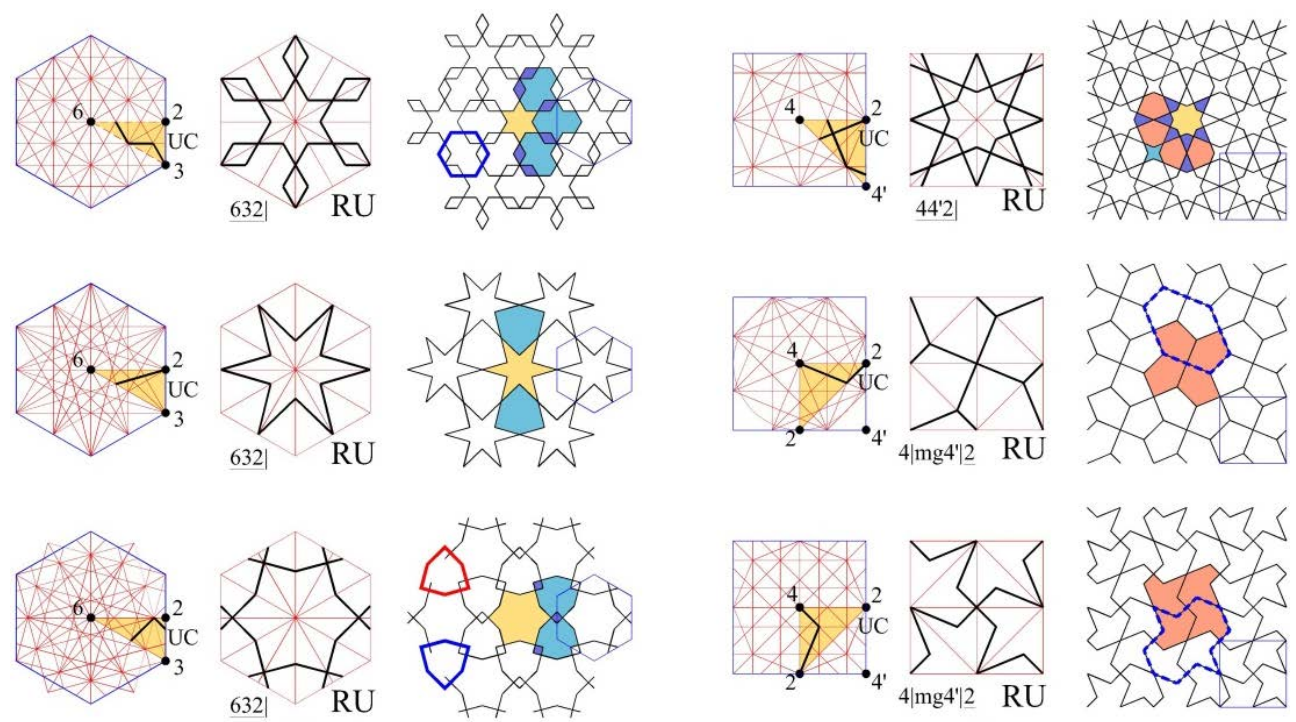

(a)
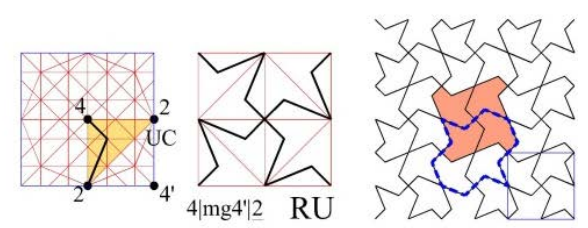

(b)

Figure 16: Geometric construction systems and modular repetition. (a) 6-fold patterns; (b) 4-fold patterns. 
16(b). Finally, the repeat unit design is tessellated again using translation in order to produce the complete patterns in the right columns of Figs 16(a) and (b) which are illustrated here in half scale for convenience.

The construction of the alternative geometric systems within repeat units, and the automation of the process of modular repetition can be easily supported by computer software as is the case in Fig. 16. The computer may even explore infinite possibilities for the selection of linear unit cell design [6], [7]. But the part of the process that demands intelligent human intervention is the selection, from among the infinite possibilities, of the ones that produce the most creative yet applicable designs. A trial and error or some kind of educated guess may unexpectedly lead to good results.

Finally, although thinking in terms of symmetry groups was most probably totally alien to the artisans or mathematicians of Antiquity and the Middle Ages, and even if the assimilation of crystallographic theories within the discipline of ornament would not have been possible for the traditional craftsman, the method of crystallographic analysis remains to be a convenient means for perception and classification of historical examples and an extremely promising method for the art of making of new designs. Emphasis in the future should be on the development of rules and guidelines that can act as design tools for guiding students, craftsmen and even industries involved in the production of new designs.

\section{REFERENCES}

[1] Grabar, O., The Formation of Islamic Art, Yale University Press: New Haven and London, 1987.

[2] Ardalan, N. \& Bakhtiar, L., The Sense of Unity: The Sufi Tradition in Persian Architecture, The University of Chicago Press: Chicago and London, 1975.

[3] Critchlow, K., Islamic Patterns: An Analytical and Cosmological Approach, Schocken Books: London, 1976.

[4] Grunbaum, B., Grunbaum, Z. \& Shephard, G.C., Symmetry in Moorish and other ornaments. Computer and Mathematics with Applications, 12B(3/4), p. 641, 1986.

[5] El-Said, I. \& Parman, A., Geometric Concepts in Islamic Art, World of Islam Festival Publishing Company Ltd: London, 1976.

[6] Dwedny, A.K., Computer recreations: imagination meets geometry in the crystalline realm of latticeworks. Scientific American, 258(6), pp. 120-123, 1988.

[7] Lalvani, H., Pattern regeneration: a focus on Islamic jalis and mosaics. The Impulse to Adorn: Studies in Traditional Architecture, ed. S. Doshi, Marg Publications: Mumbai, p. 133, 1982. 